\title{
Erratum to: Cetuximab-induced skin exanthema: prophylactic and reactive skin therapy are equally effective
}

\author{
Thomas C. Wehler · Claudine Graf • Markus Möhler • Jutta Herzog • \\ Martin R. Berger · Ines Gockel · Hauke Lang • Matthias Theobald • \\ Peter R. Galle $\cdot$ Carl C. Schimanski
}

Published online: 20 September 2013

(c) Springer-Verlag Berlin Heidelberg 2013

\section{Erratum to: J Cancer Res Clin Oncol \\ DOI 10.1007/s00432-013-1483-4}

The corresponding author would like to correct the Figs. 1 and 2 and its captions, which were published incorrectly in the original version.

The corrected figures and captions are presented below.

The online version of the original article can be found under doi:10.1007/s00432-013-1483-4.

T. C. Wehler $(\bowtie)$. C. Graf $\cdot$ M. Theobald

Third Department of Internal Medicine, Johannes Gutenberg University of Mainz, Mainz, Germany

e-mail: thomas.wehler@unimedizin-mainz.de

M. Möhler · P. R. Galle · C. C. Schimanski

First Department of Internal Medicine, Johannes Gutenberg University of Mainz, Mainz, Germany

e-mail: c.schimanski@marienhospital-darmstadt.de

J. Herzog $\cdot$ C. C. Schimanski

Department of Internal Medicine, Marienhospital Darmstadt, Martinspfad 72, 64285 Darmstadt, Germany

M. R. Berger

Toxicology and Chemotherapy Unit, German Cancer Research Center (DKFZ), Im Neuenheimer Feld 581, 69120 Heidelberg, Germany

I. Gockel · H. Lang

Department of General and Abdominal Surgery, Johannes

Gutenberg University of Mainz, Mainz, Germany 


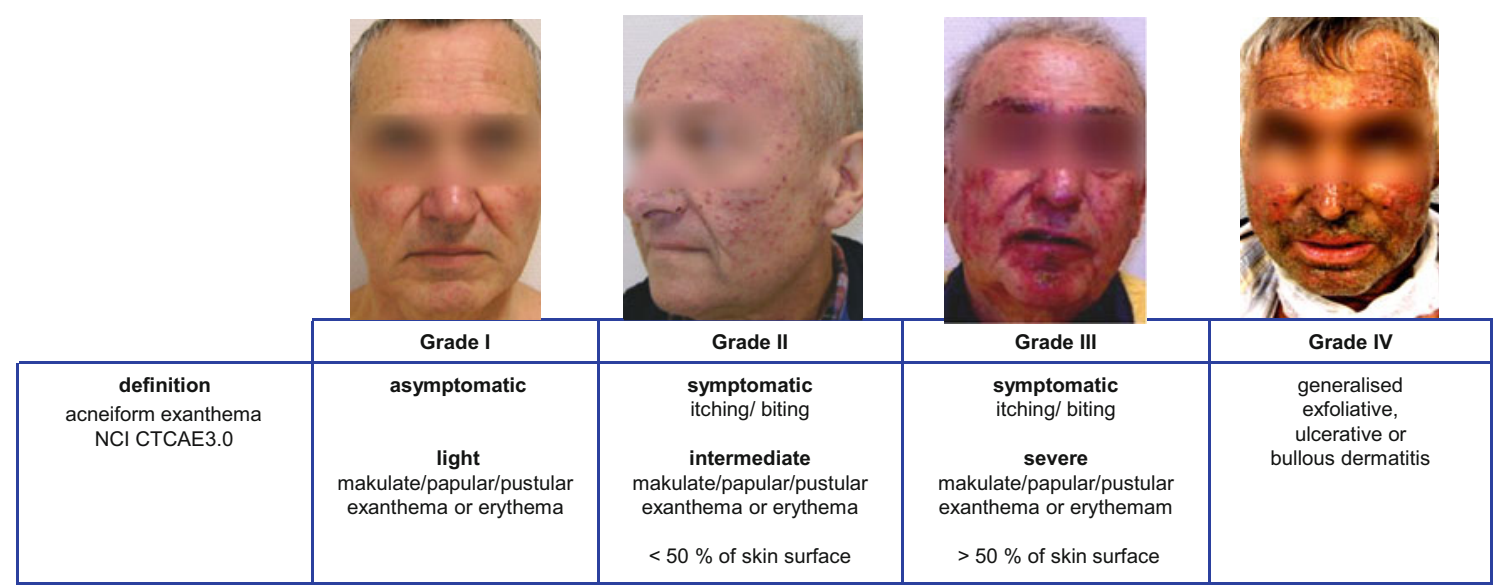

Fig. 1 Cetuximab-associated acneiform exanthema. Cetuximab-associated acneiform exanthema is classified according NCI CTCAE3.0

Fig. 2 Prophylactic treatment regimen applied to group B and reactive treatment regimen applied to group $\mathrm{C}$ prophylactic skin protocol

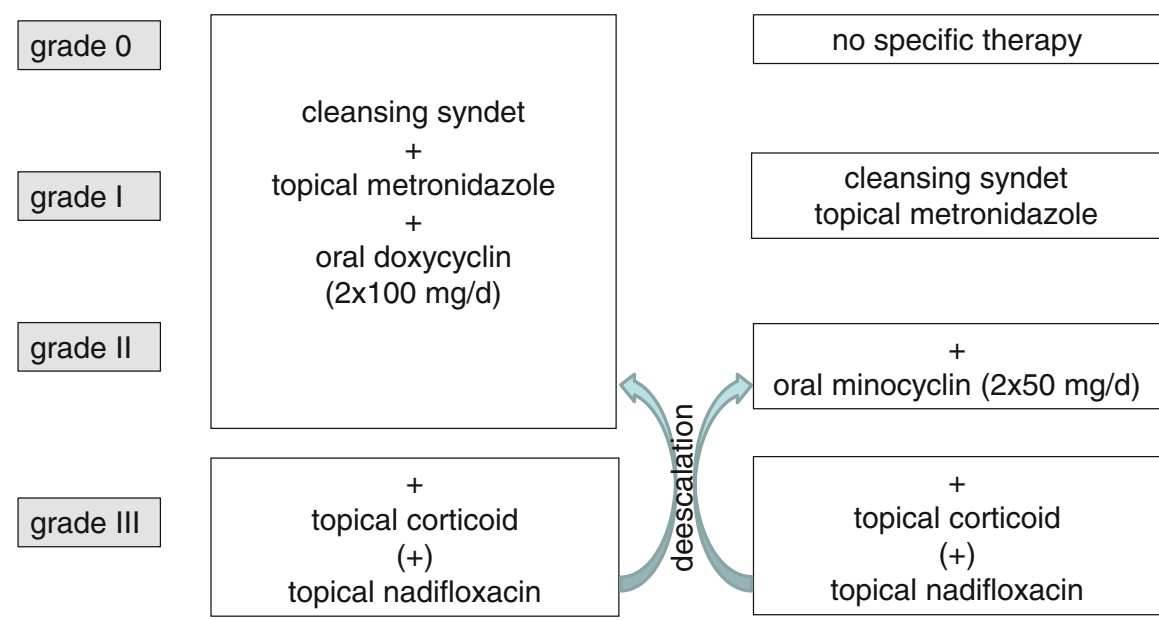

reactive skin protocol 